\title{
Intraoperative Color Doppler Ultrasound Assessment of LIMA-to-LAD Anastomoses in Off-Pump Coronary Artery Bypass Grafting
}

\author{
Rune Haaverstad, MD, PhD, Nicola Vitale, MD, PhD, Ole Tjomsland, MD, PhD, \\ Arve Tromsdal, MD, Hans Torp, PhD, and Stein O. Samstad, MD, PhD
}

St. Elisabeth Heart Center and Institute of Physiology and Biomedical Engineering, Norwegian University of Science and Technology, Trondheim, Norway

Background. Although techniques for off-pump coronary artery bypass grafting (CABG) are continually being refined, angiographic follow-up studies have indicated a higher rate of anastomoses-related stenoses than expected after traditional on-pump CABG. This study was performed to evaluate the use of intraoperative epicardial color Doppler ultrasound to quality-assess left internal mammary artery (LIMA) to left anterior descending coronary artery (LAD) anastomoses performed on the beating heart.

Methods. Twenty-four LIMA-to-LAD anastomoses were evaluated with real-time epicardial ultrasound imaging using an ultrasound transducer positioned between the paddles of the stabilizer during off-pump procedures. The length of the anastomosis $\left(D_{A}\right)$, diameters of LIMA $\left(D_{M}\right)$, LAD at the toe of the anastomosis $\left(D_{1}\right)$, and $5 \mathrm{~mm}$ distally to the anastomosis $\left(D_{2}\right)$ were measured, and the ratios between these variables were calculated. The flow velocity through the anastomoses was visualized by color Doppler coding, and flow was assessed with transit-time flowmetry.

$E^{a}$ arly graft failure is a potential life-threatening complication after coronary artery bypass grafting (CABG) [1]. During off-pump CABG (OPCAB), the distal anastomoses are performed under suboptimal conditions and the incidence of technical abnormalities of distal anastomoses requiring revision have been reported to be as high as to $9.9 \%$ [2], reflecting the need for an intraoperative method capable of detecting anastomotic errors. A reliable method for the assessment of the quality of distal anastomoses after OPCAB could reduce the frequency of graft occlusions caused by technical errors. Ideally, the method should be able to detect technical abnormalities intraoperatively, allowing instant revision of the anastomosis.

Angiography has until now been considered as the "golden standard" for assessment of anastomotic quality

Presented at the Eighth Annual Cardiothoracic Techniques and Technologies Meeting 2002, Miami Beach, FL, Jan 23-26, 2002.

Address reprint requests to Dr Haaverstad, St. Elisabeth Heart Center, University Hospital of Trondheim, Trondheim N-7018 Norway; e-mail: rune.haaverstad@medisin.ntnu.no.
Results. The epicardial color Doppler ultrasound allowed accurate assessment of the anastomoses. Twentythree $(96 \%)$ of the primary anastomoses were confirmed as patent. Mean ratios of $D_{1} / D_{2}, D_{A} / D_{2}$, and $D_{M} / D_{2}$ were $0.89 \pm 0.13,3.01 \pm 1.04$ and $1.32 \pm 0.32$, respectively. One anastomosis had a stenosis more than $50 \%$ detected by color Doppler ultrasound. After surgical revision, transittime flow increased from 22 to $40 \mathrm{~mL} / \mathrm{min}$.

Conclusions. Intraoperative color Doppler ultrasound allowed adequate imaging for quality assessment of LIMA-to-LAD anastomoses performed on the beating heart. One anastomosis was revised due to a technical error detected by epicardial color Doppler imaging. Epicardial ultrasound scanning is a valuable tool for intraoperative assessment of LIMA-to-LAD anastomoses during off-pump coronary surgery.

(Ann Thorac Surg 2002;74:S1390-4) (C) 2002 by The Society of Thoracic Surgeons

and several authors have recommended the routine use of early postoperative angiography [3]. Yet angiography is a time-consuming procedure not available in most operating rooms. The only widely recognized method of assessing graft function intraoperatively is transit-time flowmetry [4]. However, this method is of limited value because the observed flow is influenced by several hemodynamic variables such as blood pressure and vascular resistance.

Over the last 15 years several echocardiographic devices for visualization of coronary arteries and graft anastomoses have been tested in experimental models and intraoperatively in humans [5-7]. Unfortunately none of these devices have gained wide acceptance mainly because of their many technical limitations. We have previously reported our initial experience with a new linear array $10-\mathrm{MHz}$ transducer specially designed for epicardial vascular ultrasound scanning during onpump CABG [8], demonstrating its feasibility to detect and classify coronary artery stenoses. The severity score correlated significantly with the grading obtained from 
preoperative angiograms. Furthermore, ultrasound imaging allowed evaluation of the morphology of the anastomoses and a semiquantitative assessment of the flow velocity at the anastomotic site [8].

Based on our previous positive experience with onpump $C A B G$, we decided to test the device during OPCAB procedures. The present study was designed to establish a valid method of intraoperative qualityassessment of left internal mammary artery (LIMA) to left anterior descending artery (LAD) anastomoses with epicardial color Doppler ultrasound during off-pump surgery.

\section{Material and Methods}

\section{Patient Selection and Demographics}

Twenty-four consecutive patients (17 men and 7 women; $63.1 \pm 8.0$ years) scheduled for elective off-pump CABG were included. Their clinical characteristics were stable angina (CCS class II: 13, CCS class III: 11), one- or two-vessel coronary disease, and mean left ventricular ejection fraction of $0.70 \pm 0.10$. The protocol was approved by the Regional Board of Ethics in Medical Research. Informed consent was signed before inclusion. All patients received a LIMA graft anastomosed to the LAD and 13 patients received an additional graft (1 patient had a right internal mammary artery to the right coronary artery, 1 patient had a radial artery graft to the posterior descending artery, 1 patient had a saphenous vein graft to right coronary, 3 patients received a saphenous vein graft to posterior descending artery, and 2 patients a saphenous vein graft to a diagonal branch).

\section{Surgical Technique}

All operations were performed by the same surgeon (R.H.). Median sternotomy was used in all cases. After full heparinization ( $3 \mathrm{mg} / \mathrm{kg}$ ), the LIMA was harvested with its pedicle and a diluted solution of papaverine was injected intraluminally. The activated clotting time was not allowed to drift below 270 seconds. The LAD was identified and snared with a 4-0 pledgeted polypropylene suture (Prolene; Ethicon, Somerville, NJ) proximally to the incision. After 3 to 5 minutes of ischemic preconditioning, the snare was released and an epicardial stabilizer was used to immobilize the target site chosen for grafting. After the incision of LAD, an intracoronary shunt (CardioThoracic Systems, Inc, Cupertino, CA) was positioned into the vessel lumen and the coronary anastomosis was performed using a continuous $7-0$ or $8-0$ (Prolene) suture. The LIMA pedicle was secured with two epicardial stitches using 7-0 polypropylene on both sides. After assessment of the graft, heparin was reversed with protamine. The stabilizer was then removed.

\section{Epicardial Ultrasound Scanning}

After completing the LIMA-to-LAD anastomosis, with the stabilizer still in place, epicardial color Doppler scanning of the anastomosis was performed by means of a linear array $10 \mathrm{MHz}$ GE Vingmed transducer (foot-print

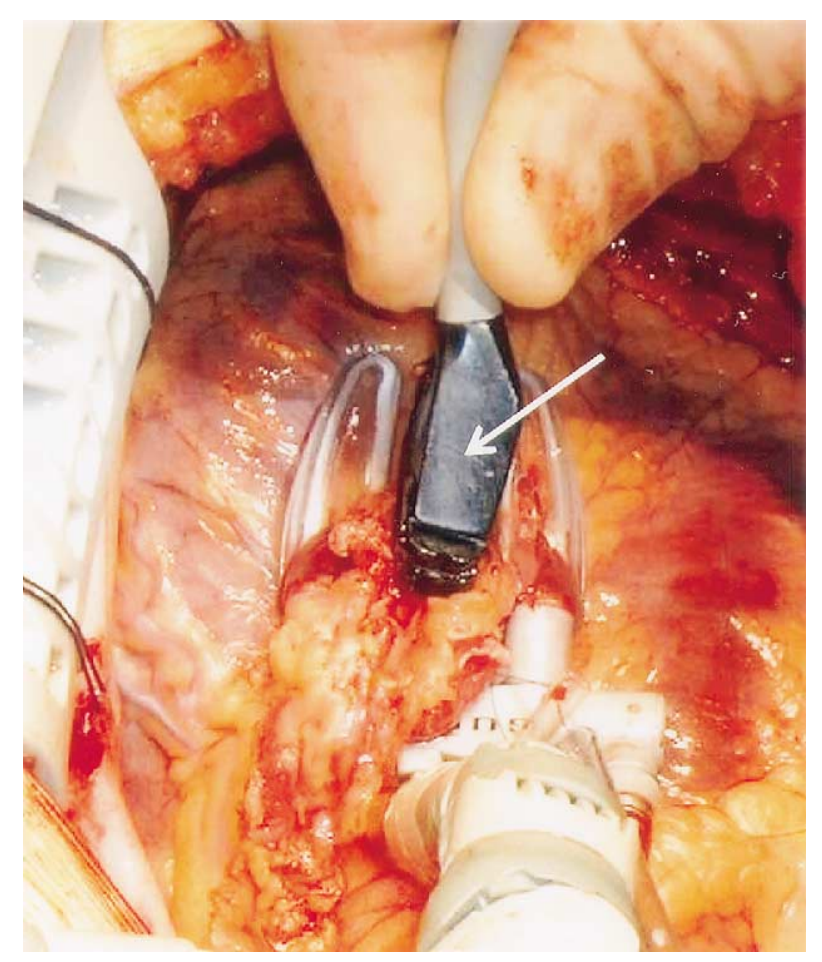

Fig 1. Intraoperative color Doppler scan of a left internal mammary artery to left anterior descending coronary artery anastomosis performed on beating heart with a $10 \mathrm{MHz}$ GE Vingmed transducer. With sterile gel as conduction medium, the ultrasound transducer (arrow) was applied epicardially between the paddles of the stabilizer.

$27.3 \times 9.6 \mathrm{~mm}$ ) connected to a GE Vingmed System FiVe echocardiography unit (GE Vingmed, Horten, Norway) [8]. With sterile gel as conduction medium, the sterilized transducer was applied directly onto the epicardium between the paddles of the stabilizer (Figure 1). Realtime ultrasound images and storage of data for later analysis were obtained within approximately 10 minutes in each patient. The ultrasound images were stored as digital data for later analysis using the Echo PAC software (GE Vingmed).

The quality of the images was rated good when both the anastomosis and the distal run-off in the coronary artery could be well visualized. Images obtained from anterior-posterior and transverse planes were used to assess the quality and patency of the anastomosis. The length of the anastomosis proper $\left(D_{A}\right)$, the diameters of LIMA $\left(D_{M}\right)$, LAD at the toe of the anastomosis $\left(D_{1}\right)$, and $5 \mathrm{~mm}$ distally to the anastomosis $\left(D_{2}\right)$ were measured (Figure 2). $D_{2}$ was defined as the reference diameter; the ratios $D_{1} / D_{2}, D_{A} / D_{2}$, and $D_{M} / D_{2}$ were calculated to visualize the geometry of the anastomosis.

\section{Transit-Time Flowmetry}

Intraoperatively, before chest closure, graft flow was measured with transit-time flowmetry (Medi-Stim Butterfly Flowmeter, Oslo, Norway) using sterile gel as conduction medium [2]. 


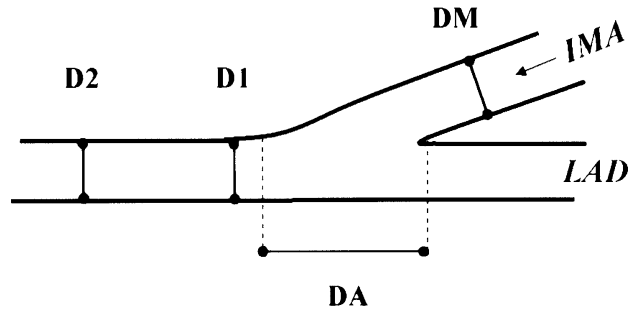

Fig 2. Details of the left internal mammary artery to left anterior descending coronary artery anastomosis, viewed in the anterior-posterior plane, assessed by epicardial color Doppler ultrasound. (IMA = internal mammary artery; $\mathrm{LAD}=$ left anterior descending coronary artery; $\mathrm{DA}=$ length of the anastomosis proper; $\mathrm{DM}=$ diameter of left internal mammary artery.)

\section{Statistical Analysis}

Data normally distributed were described as arithmetic mean and standard deviation (SD) was the measure of variability. Skewness test was used to test the distribution of the data. Skewed data are presented as median and range. A two-sampled Student's $t$ test was used to evaluate differences in normally distributed continuous data. Skewed distributed continuous data and categorical data were analyzed with the nonparametric MannWhitney test. Linear regression was used to evaluate the correlation between LAD flow rate and the $D_{1} / D_{2}$ ratio. $A$ $p$ value of less than 0.05 was considered statistically significant. Statistical analyses were performed with the NCSS program (Number Cruncher Statistical System, Kaysville, UT).

\section{Results}

No postoperative mortality or myocardial infarctions were observed. Epicardial scanning did not cause cardiac arrhythmia or hemodynamic instability in any of the patients. The transducer allowed an easy approach to the LIMA-to-LAD anastomoses.

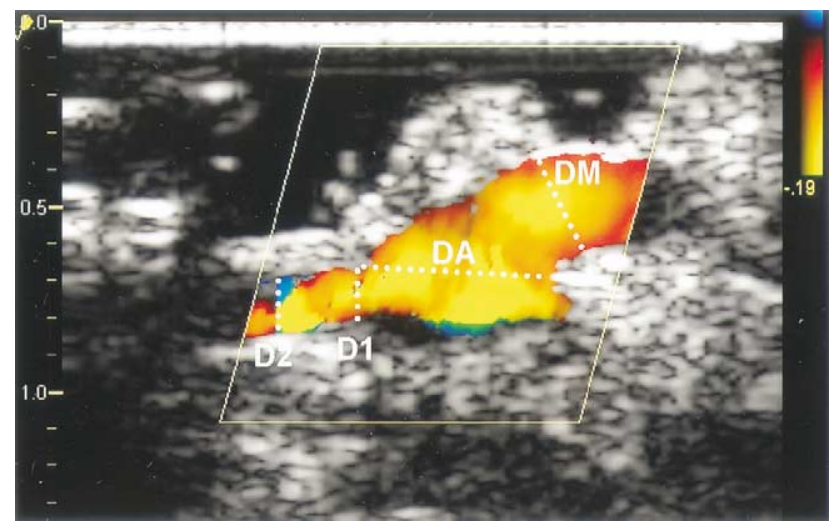

Fig 3. Intraoperative color Doppler ultrasound of a satisfactory left internal mammary artery to left anterior descending coronary artery anastomosis. There are no signs of stenosis. The D1/D2 ratio is 1 . (DA = length of the anastomosis proper; $\mathrm{DM}=$ diameter of left internal mammary artery.)
Table 1. Measurements of the LIMA-to-LAD Anastomosis Assessed by Epicardial Ultrasound Scanning in the AnteriorPosterior Plane

\begin{tabular}{lc}
\hline Measurement & Value (mean $\pm S D)$ \\
\hline$D_{1}$ & $0.15 \pm 0.05 \mathrm{~cm}$ \\
$D_{2}$ & $0.16 \pm 0.4 \mathrm{~cm}$ \\
$\mathrm{D}_{\mathrm{A}}$ & $0.49 \pm 0.15 \mathrm{~cm}$ \\
$\mathrm{D}_{\mathrm{M}}$ & $0.22 \pm 0.06 \mathrm{~cm}$ \\
$\mathrm{D}_{1} / \mathrm{D}_{2}$ & $0.89 \pm 0.13$ \\
$\mathrm{D}_{\mathrm{A}} / \mathrm{D}_{2}$ & $3.01 \pm 1.01$ \\
$\mathrm{D}_{\mathrm{M}} / \mathrm{D}_{2}$ & $1.31 \pm 0.32$ \\
\hline
\end{tabular}

No statistically significant difference was observed between $D_{1}$ and $D_{2}$ in the entire group. All other dimensions as well as ratios were significantly different from each other $\left(D_{1}\right.$ versus $D_{2}, N S ; D_{1}$ versus $D_{A}, p<0.001 ; D_{1}$ versus $\mathrm{D}_{\mathrm{M}^{\prime}} p<0.001 ; \mathrm{D}_{2}$ versus $\mathrm{D}_{\mathrm{A}^{\prime}} p<0.001 ; \mathrm{D}_{2}$ versus $\mathrm{D}_{\mathrm{M}}, p<0.001$; $\mathrm{D}_{\mathrm{A}}$ versus $\mathrm{D}_{\mathrm{M}}, p<0.001 ; \mathrm{D}_{1} / \mathrm{D}_{2}$ versus $\mathrm{D}_{\mathrm{A}} / \mathrm{D}_{2}, p<0.001 ; \mathrm{D}_{1} / \mathrm{D}_{2}$ versus $\mathrm{D}_{\mathrm{A}} / \mathrm{D}_{2}, p<0.001 ; \mathrm{D}_{\mathrm{A}} / \mathrm{D}_{2}$ versus $\left.\mathrm{D}_{\mathrm{M}} / \mathrm{D}_{2}, p<0.001\right)$.

LIMA = left internal mammary artery; $\quad$ LAD = left anterior descending coronary artery; $\quad D_{1}=$ diameter of LAD at the toe of the anastomosis; $\quad \mathrm{D}_{2}=$ diameter of LAD $5 \mathrm{~mm}$ distally to the anastomosis; $\quad \mathrm{D}_{\mathrm{A}}=$ length of the anastomosis proper; $\quad \mathrm{D}_{\mathrm{M}}=$ diameter of LIMA.

Twenty-three (96\%) anastomoses were fully patent according to color Doppler imaging (Figure 3), with median transit-time flow rate $29 \mathrm{~mL} / \mathrm{min}$ and a range from 7 to $72 \mathrm{~mL} / \mathrm{min}$. Measurements of $\mathrm{D}_{1}, \mathrm{D}_{2}, \mathrm{D}_{\mathrm{A}}$, and $\mathrm{D}_{\mathrm{M}}$, as well as the ratios $\mathrm{D}_{1} / \mathrm{D}_{2}, \mathrm{D}_{\mathrm{A}} / \mathrm{D}_{2}$, and $\mathrm{D}_{\mathrm{M}} / \mathrm{D}_{2}$ are presented in Table 1 . No statistically significant difference was observed between $D_{1}$ and $D_{2}$ in the entire group. All other dimensions and ratios were significantly different from each other (Table 1). No significant correlation was observed between LAD flow and the $D_{1} / D_{2}$ ratio $\left(r^{2}=0.05, p=0.1\right)$. Nine anastomoses $(38 \%)$ with $\mathrm{D}_{1} / \mathrm{D}_{2}$ less than 0.9 (mean $0.79 \pm 0.14$ ) had a minor intraluminal echo-dense protrusion observed at the toe.

To assess whether a decreased $D_{1} / D_{2}$ ratio could limit graft flow, we decided to compare LIMA-to-LAD flow between patients with $D_{1} / D_{2}$ ratio below the mean value (0.89) with the graft flow observed in patients with a $D_{1} / D_{2}$ above the mean value. No difference in LIMA-toLAD flow was observed between $D_{1} / D_{2}$ more than 0.89 versus $\mathrm{D}_{1} / \mathrm{D}_{2}$ less than 0.89 [27 (16 to 69$) \mathrm{mL} / \mathrm{min}$ versus 22 (7 to 72$) \mathrm{mL} / \mathrm{min}$, respectively; $p=0.57]$. In one anastomosis the $D_{1} / D_{2}$ ratio was 0.43 , and a large echodense protrusion as well as an increased flow velocity were observed in the color Doppler image on longitudinal and transverse views. Despite a transit-time flow rate of $22 \mathrm{~mL} / \mathrm{min}$, the ultrasound findings were suggestive of a more than $50 \%$ stenosis at the toe of the anastomosis. Revision of the anastomosis revealed an intimal flap in the LAD. After revision, the transit-time flow rate increased to $40 \mathrm{~mL} / \mathrm{min}$.

\section{Comment}

The present study demonstrates that epicardial color Doppler scanning with a new $10 \mathrm{MHz}$ GE Vingmed transducer provides satisfactory visualization of LIMAto-LAD anastomoses, allowing accurate measurements 
of the length of the anastomosis proper $\left(D_{A}\right)$, diameters of LIMA $\left(D_{M}\right)$, LAD at the toe of the anastomosis $\left(D_{1}\right)$, and $5 \mathrm{~mm}$ distally in the downstream LAD $\left(\mathrm{D}_{2}\right)$. Furthermore, we were able to detect a stenosis in the anastomosis presenting with a normal transit-time flow pattern. Epicardial scanning did not cause cardiac arrhythmia or hemodynamic instability in any of the patients.

A patent LIMA-to-LAD graft is the single most important determinant of long-term and event-free survival and imperfect graft anastomoses have a poor long-term patency after CABG $[9,10]$. Epicardial color Doppler scanning allows an accurate assessment of the LIMA-toLAD anastomoses and provides three important pieces of information: visualization of the anastomosis and its components (LAD and LIMA); measurement of the length of the anastomosis proper as well as the diameters of the LAD downstream and the LIMA; and color Doppler assessment of blood flow velocity at the anastomotic site. The necessary measurements were obtained easily without risk of any complications, are not time consuming, and provide important information about the geometry and the flow pattern of the anastomosis.

Minor intraluminal protrusions at the toe were observed in nine of the anastomoses with a $D_{1} / D_{2}$ ratio of less than 0.9. Incongruence or a slight bulging at the edges of the vessel walls where the graft and the LAD are joined together may explain these findings. However, no major changes in blood velocity were observed at these sites. Anastomoses may develop temporary edema, hematoma, and spasm, and some angiographic follow-up studies have suggested that some FitzGibbon B lesions seen with immediate or early postoperative angiography have spontaneously resolved at later coronary angiography $[9,11]$. Transit-time flowmetry has been widely used to evaluate the quality of distal anastomoses after CABG. However, the lowest acceptable flow value in the LIMA graft has not been not defined and graft revision has been advised when flow ranges from 5 to $20 \mathrm{~mL} / \mathrm{min}[4,12]$. This wide range indicates that the mean graft flow alone is not a reliable measurement for graft assessment, unless the stenosis is more than $75 \%$ to $90 \%[13,14]$. The only anastomosis with a marked stenosis observed in the present study was detected by epicardial imaging in a patient with a LIMA transit-time flow rate of $22 \mathrm{~mL} / \mathrm{min}$. During surgical revision, an intimal flap at the toe of the anastomosis was detected. After revision LIMA flow increased to $40 \mathrm{~mL} / \mathrm{min}$. Intimal flap is a well-known technical failure of the anastomosis, along with thrombosis, stenosis at the heel, coronary dissection, LIMA dissection, and vessel stenosis distal to the graft $[4,15,16]$. The epicardial color Doppler scanning technique used in the present study may be considered the only method able to identify all these problems, and may accordingly offer an important tool for detecting anastomotic errors leading to early graft failures.

Concern has been raised regarding the use of temporary snares and shunts because of the potential risk of endothelium alteration [17]. We were not able to detect any LAD lesion caused by snares or intraluminal shunts with intraoperative epicardial color Doppler scanning.
However, our patient cohort will undergo angiographic follow-up to evaluate whether stenosis at these sites develop over time.

Previous experimental and clinical studies have demonstrated that the assessment of the technical adequacy of bypass grafts may be enhanced by the use of epicardial echocardiography $[5-7,18,19]$. The main reason why epicardial echocardiography has not gained more popularity is the large size of the ultrasonic probe, allowing only the visualization of LAD, diagonals, and proximal right coronary artery [5-7, 18, 19]. Coronary branches located laterally or at the back of the heart could be scanned only during CPB because the dislocation of the heart caused hemodynamic instability. When performing OPCAB through a median sternotomy, the heart is exposed with pericardial stitches and modern stabilizers, allowing epicardial scanning of coronary vessels also on the inferior and lateral walls. In the present study we emphasized quality assessment of LIMA-to-LAD anastomosis. However, the design of the transducer allows epicardial imaging of all areas, and we plan to use it for assessing all distal anastomoses with the stabilizer still in place in future studies.

The early-generation transducers were able to provide only tissue images. In this study the color Doppler modality was obtained as a semiquantitative measure. Although color Doppler imaging may be given as an absolute velocity with the device used in our study, this measurement is still not reliable intraoperatively in a clinical setting because of difficulties in adjusting for the angle created between the transducer and the blood flow within the vessels. The latter problem points out that the technology of intraoperative color Doppler imaging in off-pump coronary surgery still needs further development, and the prognostic value of graft patency has yet to be evaluated by follow-up angiography. The introduction of this new transducer for epicardial imaging of small vessels with color Doppler features may enhance the widespread use of epicardial scanning in coronary artery surgery.

\section{Conclusions}

The present study demonstrated that epicardial color Doppler scanning with the new $10 \mathrm{MHz}$ GE Vingmed transducer provides visualization of LIMA-to-LAD anastomoses that allows accurate measurements of the anastomosis. All these measurements are easily obtained without risk of any complications, are not timeconsuming, and provide important information about the geometry and flow pattern of the anastomosis. The introduction of this new transducer for epicardial imaging of small vessels with color Doppler features may enhance the widespread use of epicardial scanning in coronary artery surgery.

\section{References}

1. Rasmussen C, Thiis JJ, Clemmensen $P$, et al. Significance and management of early graft failure after coronary artery bypass grafting: feasibility and results of acute angiography 
and re-re-vascularization. Eur J Cardiothorac Surg 1997;12: $847-52$.

2. D'Ancona G, Karamanoukian HL, Salerno TA, Schmid S, Bergsland J. Flow measurement in coronary surgery. Heart Surg Forum 1999;2:121-4.

3. Izzat MB, Khaw KS, Atassi W, Yim AP, Wan S, El Zufari MH. Routine intraoperative angiography improves the early patency of coronary grafts performed on the beating heart. Chest 1999;115:987-90.

4. D'Ancona G, Karamanoukian HL, Ricci M, Schmid S, Bergsland J, Salerno TA. Graft revision after transit time flow measurement in off-pump coronary artery bypass grafting. Eur J Cardiothorac Surg 2000;17:287-93.

5. Hiratzka LF, McPherson DD, Brandt B III, et al. The role of intraoperative high-frequency epicardial echocardiography during coronary artery revascularization. Circulation 1987; 76:V33-8.

6. Ishikura F, Matsuwaka R, Sakakibara T, Sakata Y, Hirayama A, Kodama K. Clinical application of power Doppler imaging to visualize coronary arteries in human beings. J Am Soc Echocardiogr 1998;11:219-27.

7. Kerber RE, McPherson DD, Sirna SJ, Ross A, Marcus ML. What have we learned about coronary artery disease from high-frequency epicardial echocardiography? Int J Card Imaging 1989;4:169-76.

8. Haaverstad R, Vitale N, Williams RI, Fraser AG. Epicardial colour Doppler scanning of coronary artery stenosis and graft anastomoses. Scan Cardiovasc J 2002;36:95-9.

9. Gill IS, FitzGibbon GM, Higginson LA, Valji A, Keon WJ. Minimally invasive coronary artery bypass: a series with early qualitative angiographic follow-up. Ann Thorac Surg 1997:64:710-4.

10. Lytle BW, Loop FD, Cosgrove DM, Ratliff NB, Easley K, Taylor PC. Long-term (5 to 12 years) serial studies of internal mammary artery and saphenous vein coronary bypass grafts. J Thorac Cardiovasc Surg 1985;89:248-58.

11. Hol PK, Fosse E, Mork BE, et al. Graft control by transit time flow measurement and intraoperative angiography in coronary artery bypass surgery. Heart Surg Forum 2001;4:254-7.

12. Barnea $\mathrm{O}$, Santamore WP. Intraoperative monitoring of IMA flow: what does it mean? Ann Thorac Surg 1997;63:S12-7.

13. Jaber SF, Koenig SC, BhaskerRao B, VanHimbergen DJ, Spence PA. Can visual assessment of flow waveform morphology detect anastomotic error in off-pump coronary artery bypass grafting? Eur J Cardiothorac Surg 1998;14:476-9.

14. VanHimbergen DJ, Koenig SC, Jaber SF, Cerrito PB, Spence PA. A review of transit-time flow measurement for assessing graft patency. Heart Surg Forum 1999;2:226-9.

15. Pagni S, Qaqish NK, Senior DG, Spence PA. Anastomotic complications in minimally invasive coronary bypass grafting. Ann Thorac Surg 1997;63:S64-7.

16. Takami $Y$, Ina H. Relation of intraoperative flow measurement with postoperative quantitative angiographic assessment of coronary artery bypass grafting. Ann Thorac Surg 2001;72:1270-4.

17. Grubitzsch H, Ansorge K, Wollert HG, Eckel L. Stunned myocardium after off-pump coronary artery bypass grafting. Ann Thorac Surg 2001;71:352-5.

18. McPherson DD, Armstrong M, Rose E, et al. High frequency epicardial echocardiography for coronary artery evaluation: in vitro and in vivo validation of arterial lumen and wall thickness measurements. J Am Coll Cardiol 1986;8:600-6.

19. Sakakibara T, Matsuwaka R, Ishikura F, Mitsuno M, Yagura A, Kodama K. Intraoperative real-time visualization of coronary arteries by means of power Doppler echocardiography: preliminary experience. J Thorac Cardiovasc Surg 1997; 113:605-6. 